\title{
Speech Synthesis of Arabic Vocabularies
}

Enaam Ghanim Saeed

enaamghanim@uomosul.edu.iq

College of Computer Sciences and Mathematics

University of Mosul, Mosul, Iraq

\section{Received on:5/1/2011}

\section{ABSTRACT}

Accepted on:16/3/2011

In this paper, system is presented for speech synthesis of Arabic vocabularies written with diacritics, graphemes transfer to allophones by applying the phonological rules. The system depend on phonetics units series every unite consists of one ore more phonetic character. We create linguistic group from Arabic phonemes and a group of phonological rules and special and unusual cases by designing special function for this purpose. We use (visual basic v.6) to design this system. Evaluation of the system was undertaken to assess the accuracy on word and sentence levels. The results showed high perception levels about $84 \%$.

Keywords: speech phonetics,synthesis

$$
\begin{aligned}
& \text { تركيب الكلام من المفردات العربية } \\
& \text { إنعام غانم سعيد } \\
& \text { كلية علوم الحاسوب والرياضيات، جامعة الدوصل }
\end{aligned}
$$

تاريخ قبول البحث: 16 / 3 / 2011

تاريخ استلام البحث: 5 / 1 / 2011

\section{الملخص}

في هذا البحث، تم تصميم نظام لتركيب الكلام من المفردات العربية المشكلة من رموز كتابية إلى رموز

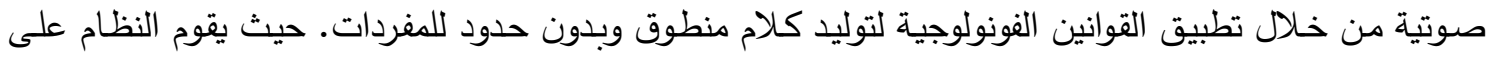
مبدأ تسلسل الوحدات الصوتية أي كل وحدة صوتية تتكون من حرف صوتي واحد أو أكثر من حرف مدمج في نفس الوحدة ويكون مشكل بالحركات. حيث تم تكوين مجموعة لغوية تتألف من الوحدات الصوتية العربية بصوت منوتيه

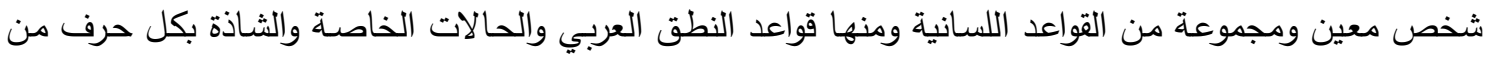

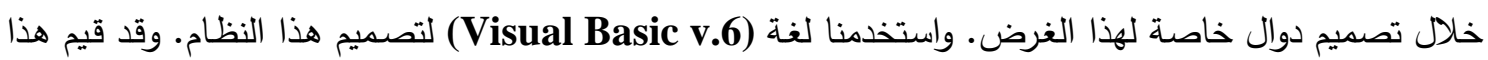

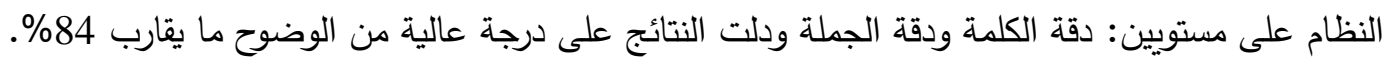
الكلمات المفتاحية: كلام ,وحدة صوتية,تركيب

تركيب الكـلام هو التوليد الأوتومـاتيكي للكـلام (الموجـات الأوكستية) مـن المفردات اللغويـة. الاختلاف

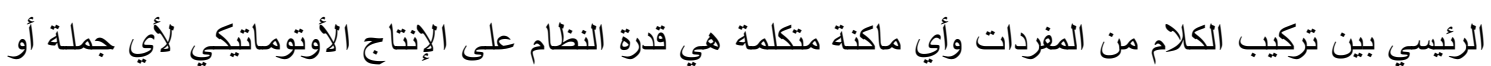
كلمة جديدة [4].

فاللغـة العربيـة تتمتع بقدرات صـوتية هائلـة تفوق مـا تحملـه اللغـات الأخرى. ممـا يتطلب وضـع أنظمـة

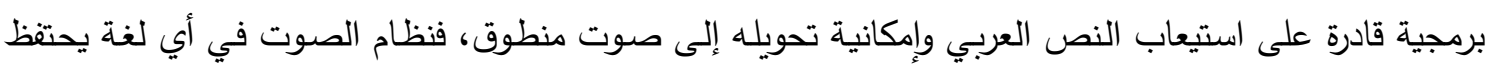

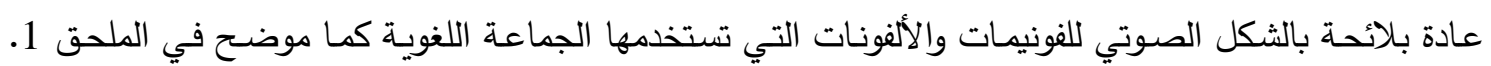
ولهذا اعتمدنا في تصميم هذا النظام على الفونيمات والألفونات الخاصة باللغة العربية [2].

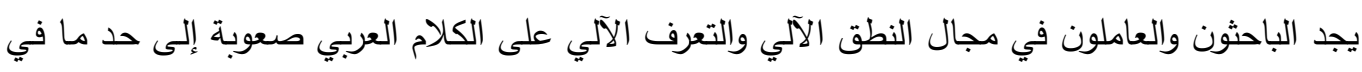
التعامل مع النص العربي وذلك من حيث تحويل رموزه المكتوبة إلى رموز صوتية. رغم إن العربية تكاد تكون أقدم 
لغة حددت أصواتها ودون نظامها وقوانينها الفونولوجية (قبل أكثر من 12 قرن) إلا انه لم توضع هذه الخصائص

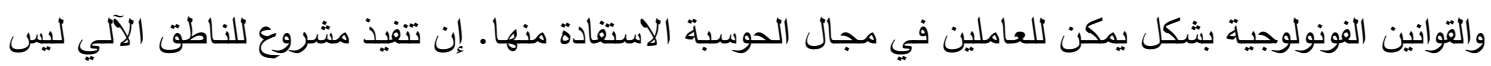
بالأمر اليسير، مثله في ذلك مثل تتفيذ نفس النظام لأية لغة أخرى ولو أن الخصائص اللسانية العربية من اقلها

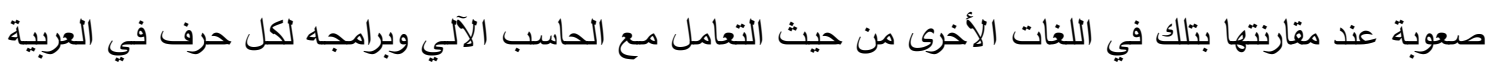

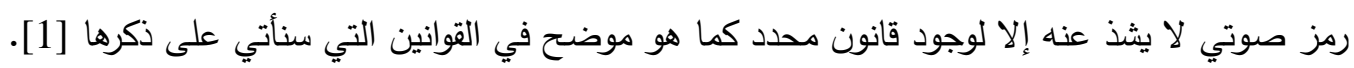
ولقد قدم الباحثون العرب عدد من البحوث في هذا المجال ففي عام 2005 صمم نظام تركيب الكلام من فن فئن النص بالاعتماد على ثنائيات الأصوات واستخدام تقنيات PSOLA [5] . وفي 2006 صمم نظام إضـافة العاطفة (كالحزن، والفرح، والغضب، والخوف) مع الكلام المنتج بحسب الموقف المرافق للحالة [6]. واعتمد باحثون آخرون في عام 2009 على الثبكات العصبية في تحليل النص وإنتاج الكلام [3]. وفي 2010 قدم بحث في تحويل النص العربي إلى كلام بالاعتماد على ثنائيات الأصوات واستخدام MARRY TTS [4].

\section{2- الههف من البحث:}

مـع التقدم السريع في تكنولوجية المعلومات والاتصـالات تطلب تقديم أنظمـة حاسوب توفر للمستخدمين

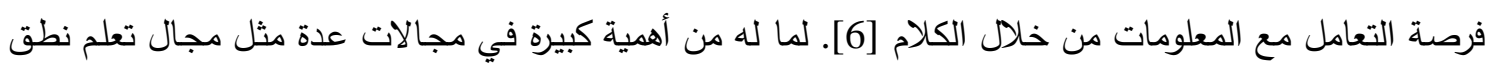

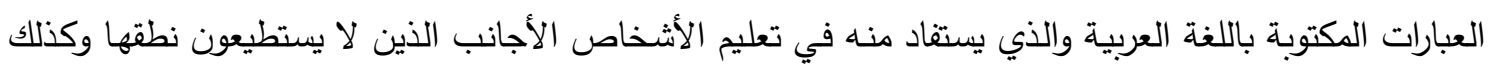

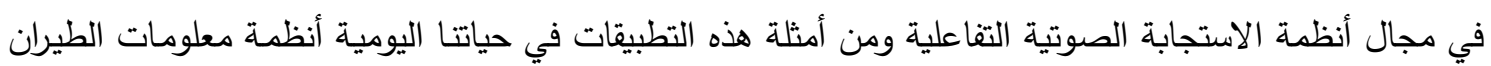

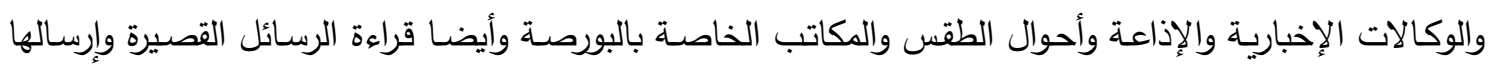

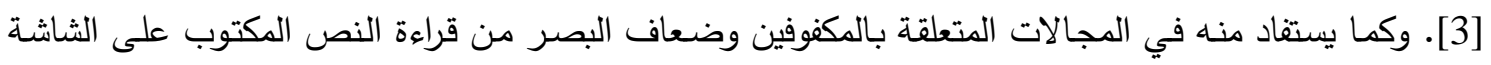

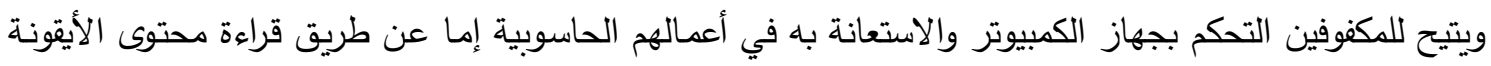

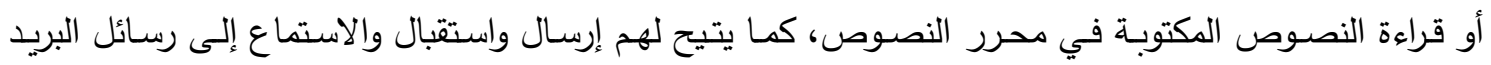

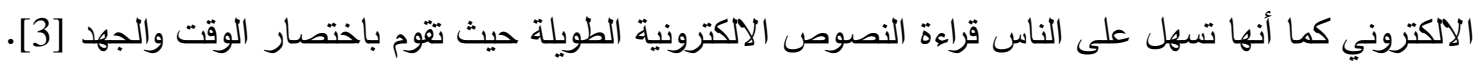
ولأهمية الموضوع صممت وطورت أنظمة تركيب الكلام للغات عدة مثل الانكليزية والفرنسية والاسبانية وغيرها ومن جهة أخرى كانت فرصة اللغة العربية قليلة مقارنة باللغات أخرى بنفس الأهمية. ونتيجة لذلك قدمنا هذا

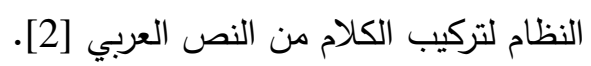

3- 3 القوانين الفونولوجية: [2]

لتحويـل المفردات المكتوبـة باللغـة العربيـة إلى كـلام منطوق فــن الضـروري استخدام وتطبيق القوانين

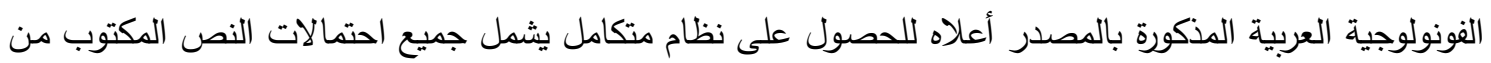
جمل اعتيادية أو شاذة ومن ضمن هذه القوانين الإقلاب والإخفاء والإدغام ... الخ. وفيما يلي القوانين الفونولوجية التي تم تطبيقها في هذا البحث:

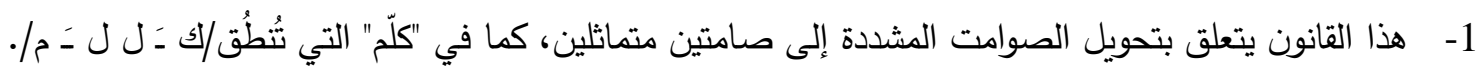

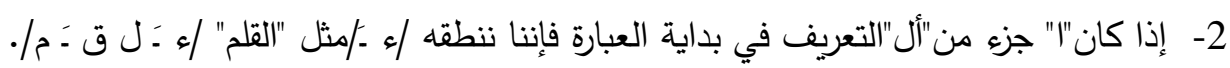

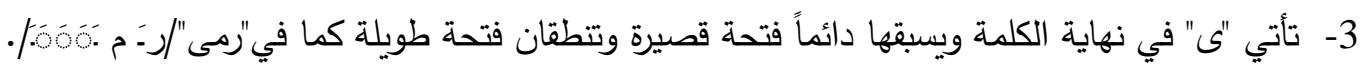

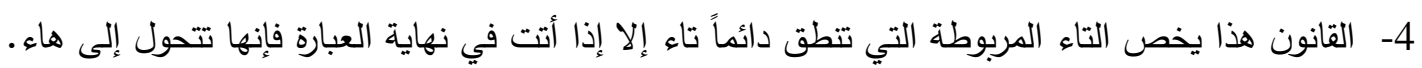




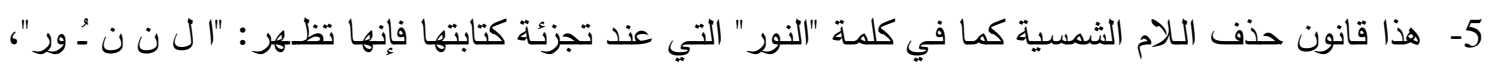

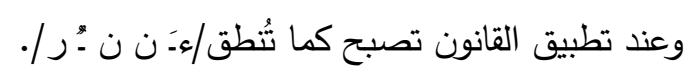

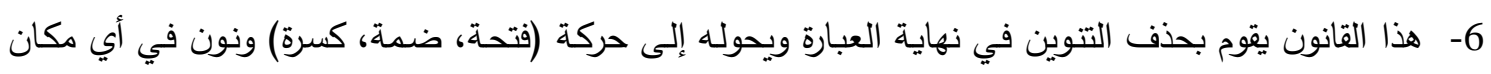

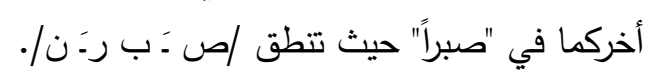

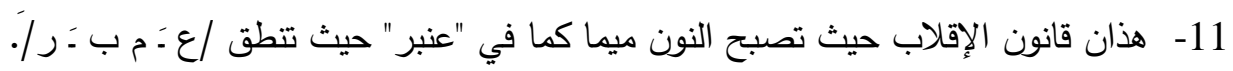

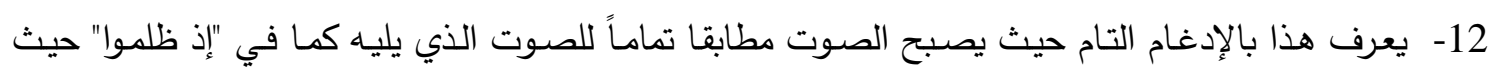

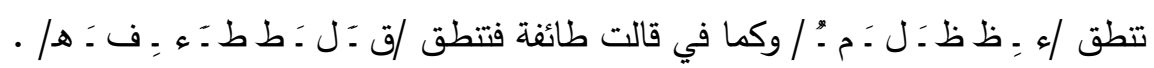

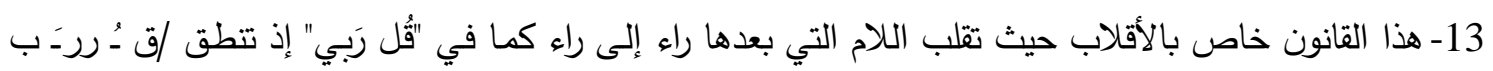

14- هذا قانون الترقيق والتفخيم للراء، فالراء مرققة إذا كانت مكسورة أو كانت ساكنة مسبوقة بكسرة، ومفخمة فيما عدا ذلك

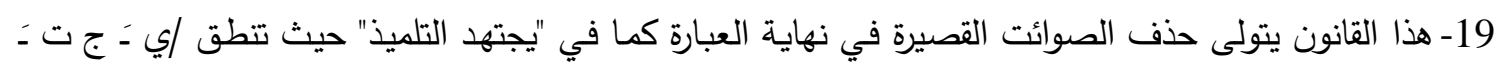

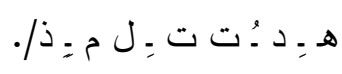

ملاحظة: تسلسل القوانين في هذا البحث ذكرت كما جاءت في المصدر. 4- - الموارد التي تطلبها النظام:

أن عدد الموارد التي يحتاجها النظام محدودة و متمثلة بما يلي:

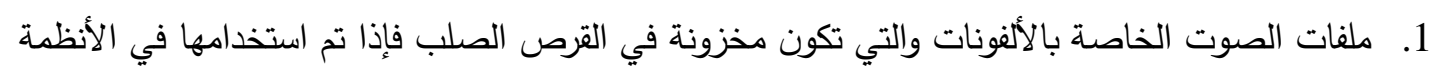

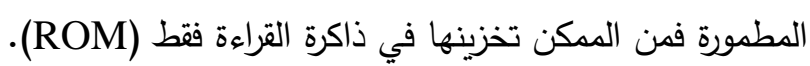

2. لوحة المفاتيح (Key Board) الخاصة بالحاسب الثخصي والتي من الممكن بنائها عن طريق استخدام

المسيطر الدقيق (Micro Controller) أو (FPGA).

3. شاشة العرض (Monitor) والتي من المككن الاستعاضة عنها بثاشة عرض البلورات السائلة (LCD) وبناء البرنامج الخاص بملائمة الثاشة.

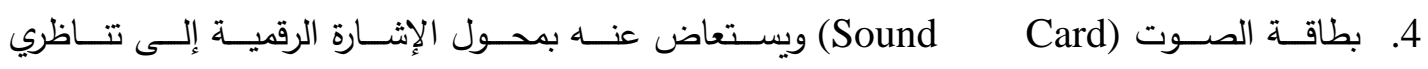
للحصول على إثارة الصوت التتاظرية. (DAC: Digital to Analog Converter) 5. مكبر صوت (Audio Power Amplifier) ذو قدرة معينة وحسب متطلبات المستخدم.

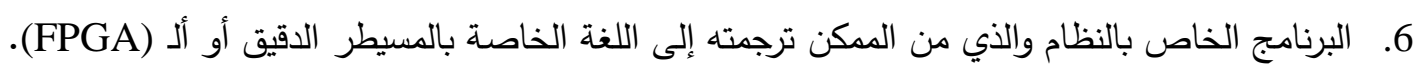
7. مجز القدرة لتجهيز مكونات النظام المطمور بالجهود والتيارات المطلوبة. 5- المراحل التي تم القيام بها لتحويل المفردات العربية إلى كلام:

قمنا بإتباع الخطوات التالية لدعالجة المفردات وتحويلها إلى كلام منطوق للحصول على نتائج جيدة جدا ومتكاملة والثكل (7) يمثل المخطط الانسيابي المبني والخاص بالبحث. وفيما يلي هذه الخطوات: 
في هذه المرحلة اعتمدنا على إدخال المفردات المشكلة لتوفير جميع الرموز الكتابية للأصسوات اللغويـة

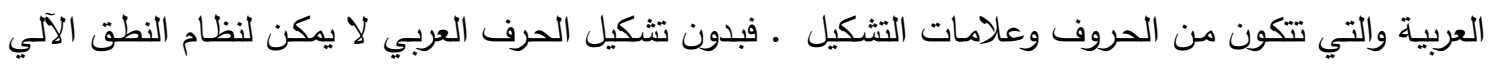

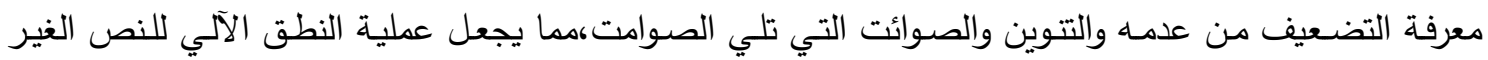

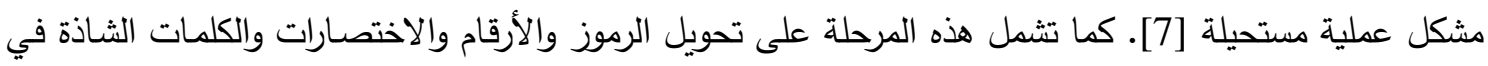

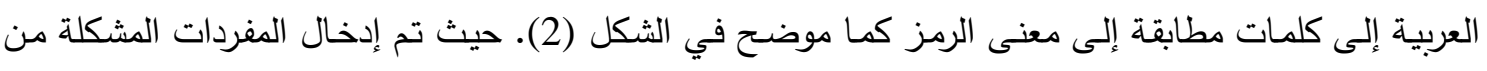

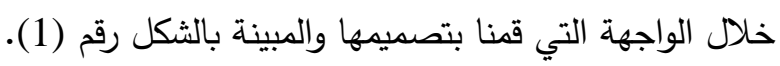

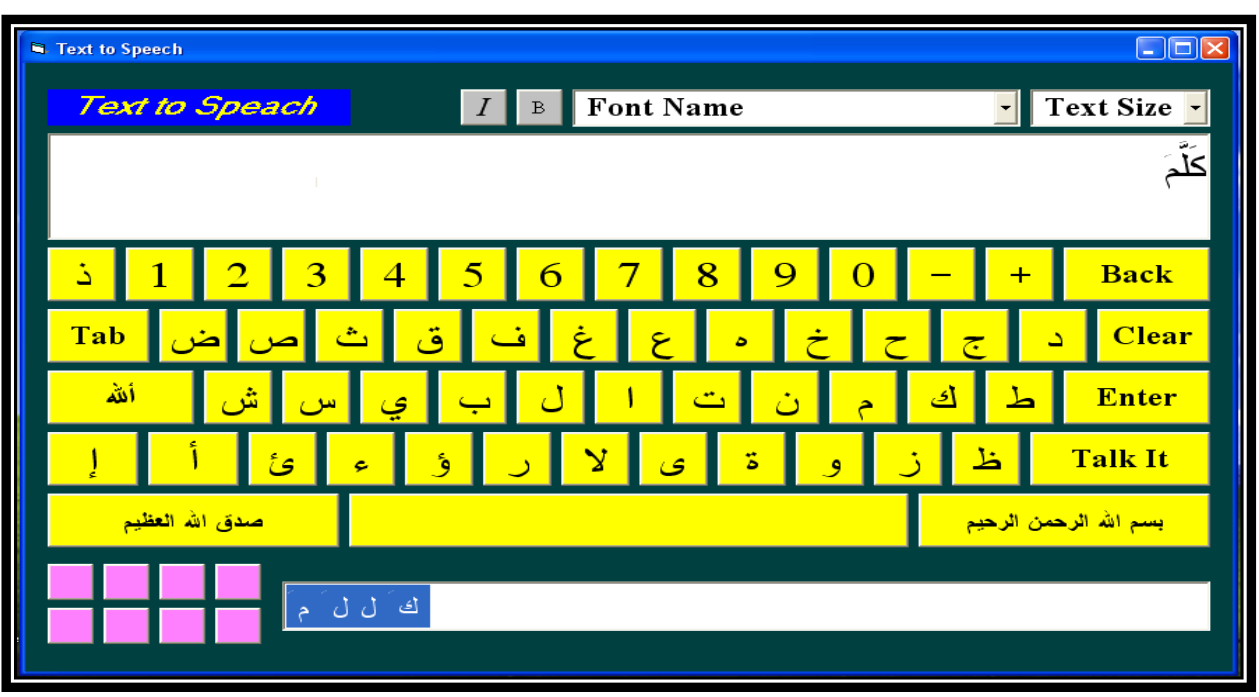

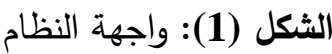

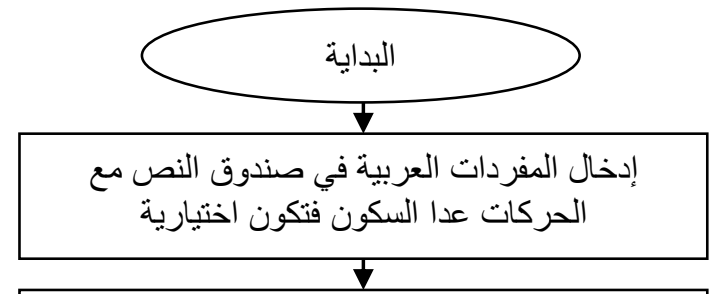

قر اءة النص العربي وخزنه في سلسلة (String)

7

تحويل السلسلة إلى مصفوفة الأحرف الأحادية الأبعاد

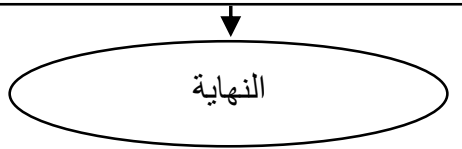

الثكل (2): المخطط الانسيابي لإدخال النص

2-5 تحويل الحرف العربي والحركة التي عليه إلى رمز صوتي يسمى فونيم

Acoustic ( القصد من الرموز هنا هو تعريف نطق كل حرف بحيث يمكن تحديد خصائصه الأكوستية

(Characteristics ومـن ثم توليدها وهذه المرحلـة التي تثـتمل على نطق الحروف والرمـوز العربيـة،بما فيهـا 
التثكيل في جميع المواقع. وللحصول على فونيمات اللغة العربية بوضوح تم اختيار متحدث يتمتع بجودة الصوت وتكون مخارج الأحرف العربية لديه واضحة وصحيحة، وحيث انـه بعض الأحرف تكون من الصعوبة تسجيلها

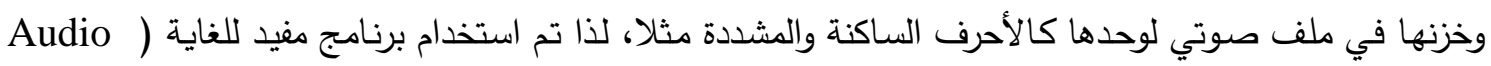
(Editor Deluxe وصحيح وكما موضح في الثكل (3) والتي تمثل حرف الباء ساكنا مقطوعاً من كلمة ما بسبب صعوبة نطق حرف التف الباء ساكنا كما ذكرنا والثكل (4) يمثل حرف الباء مضموماً.

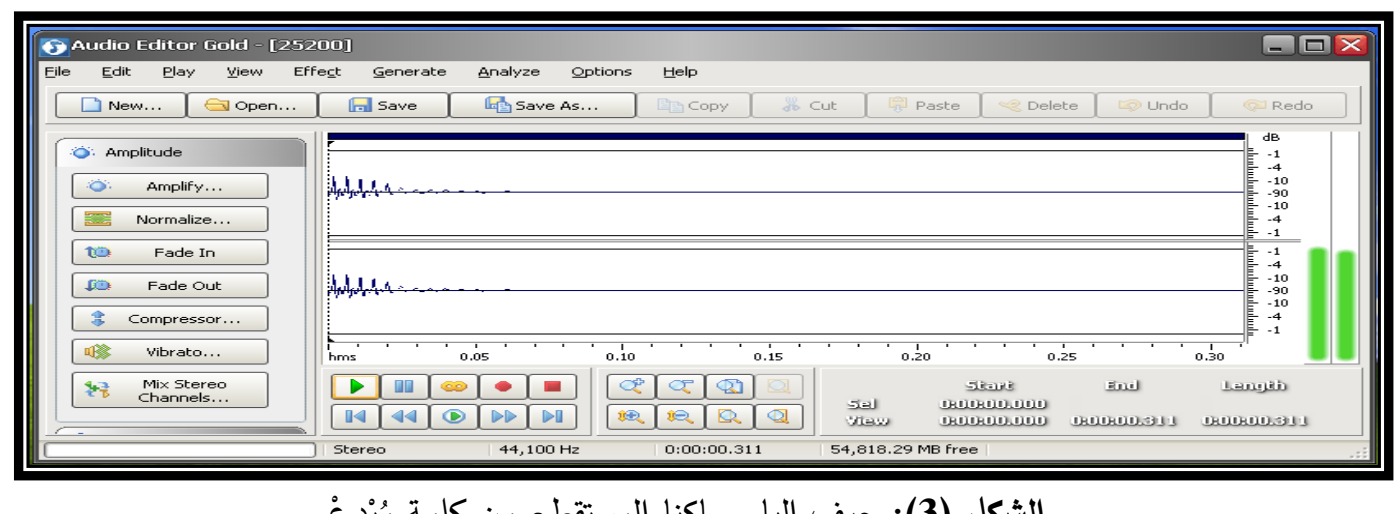

الشكل (3): حرف الباء ساكنا المستقطع من كلمة مُبْدِغْ

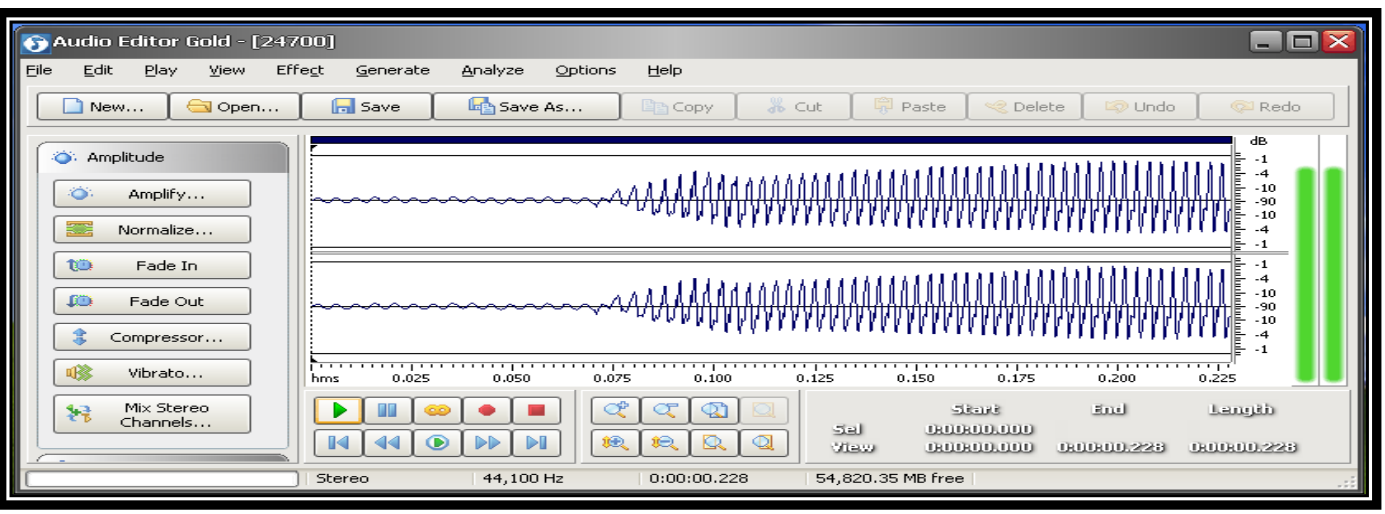

الثكل (4): حرف الباء مضموما

3-5 وضع القواعد الفونولوجية التحويلية حيث تتولى القواعد في هذه المرحلة تحويل الفونيمات إلى الوفونات الفونولوجية للأصوات (الفونيمات) على بعضها البعض وتحديد الصوت الذي يتم إخراجه في نهاية المطاف. ويندرج تحت هذه القواعد تأثيرات الأصوات المتجاورة وما تجاوزها إلى الأصوات التي بعدها وذلك من خلال تطبيق القوانين الفونولوجية الأنفة الذكر جميعها وبالتسلسل

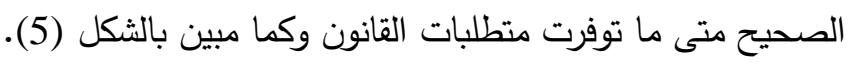




\section{4-5 ربط الألفونات ببعضها بحيث يتوفز الانتقال الأكوستي}

إن الإثارة الأكوستية لا تتتقل فجأة من صوت لغوي ما إلى صوت أخر ، إذ لابد من فترة للانتقال بين

الأصوات اللغوية. وهذا يعطي الكلام خاصية الوضوح بحيث يمكن للسامع فهم ما يقال كما في الثكل (6).

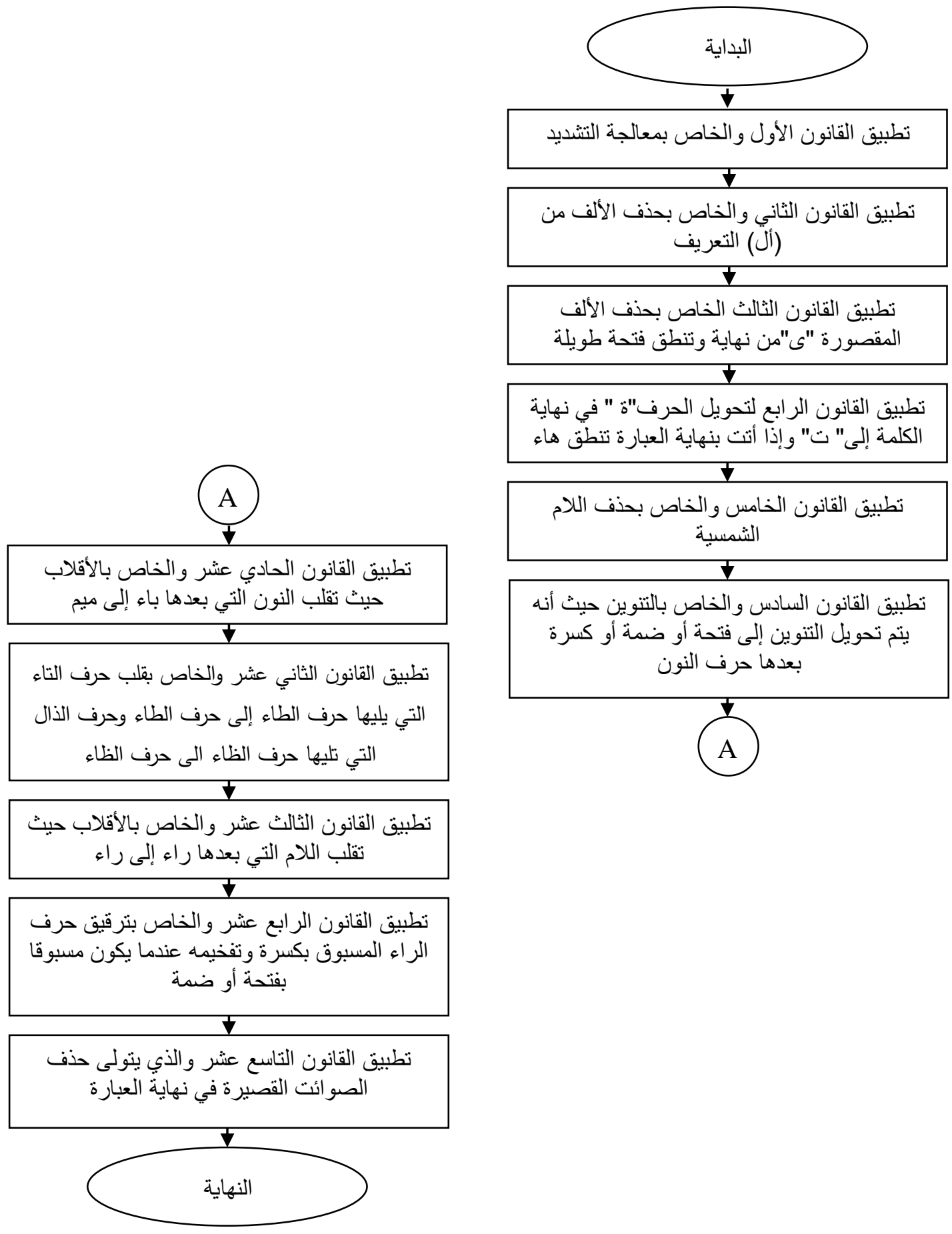

الثكل (5): المخطط الانسيابي لتطبيق القواعد الفونولوجية 


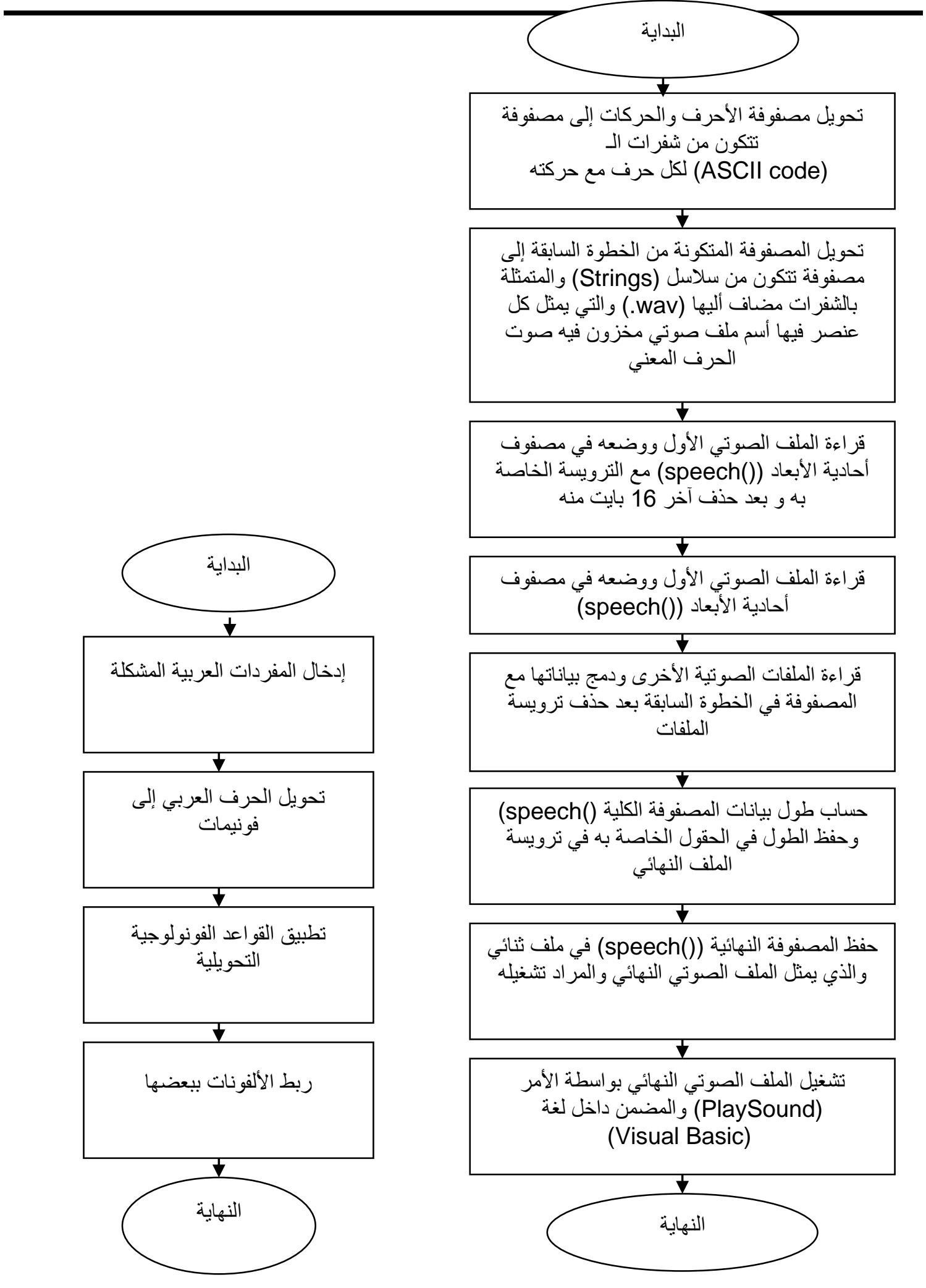

الثكل (7): المخطط الانسيابي العام للنظام

الثكل (6): المخطط الانسيابي لتطبيق القواعد الفونولوجية

تم بناء نظام متكامل لنطق المفردات (كلمة، جملة) المكتوبـة باللغة العربية وبعدد من الألفونـات يعتبر

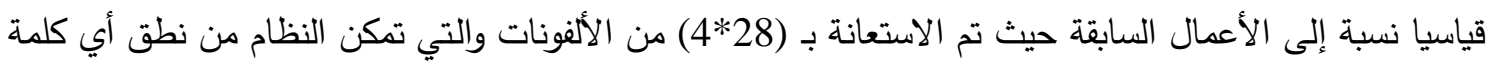
من كلمات اللغة العربية. 
تطبيق أكبر عدد من قوانين الفونولوجية العربية وكذلك الاستفادة من الكتابة العروضية التي تستخدم من قبل الشعراء الذين يكتبون الثعر بالاعتماد على الأوزان الخاصـة ببحور الثعر العربي والتي سهلت كثيرا عملية

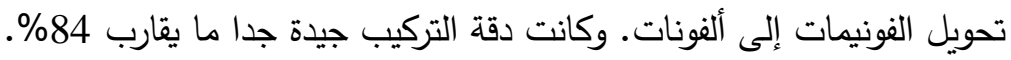
الخطوات اللازمة لتحويل الكتابة النصية إلى منطوقة:

1. إدخال النص مع الحركات كسلسلة من الأحرف (String of characters): كَتَبَ الطَالِبُِ الوَاجِبَ

2. تحويل السلسلة الحرفية إلى مصفوفة أحادية تضم الحروف والحركات وكما موضح أدناه:

\begin{tabular}{|c|c|c|c|c|c|c|c|c|c|c|c|c|c|c|c|c|c|c|c|c|c|}
\hline س & נ & 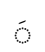 & د & \multicolumn{2}{|l|}{ ل } & 8 & ب & @ & ل & 1 & 8 & ط & J & 1 & 0 & ب & 8 & ت & & 5 & ك \\
\hline
\end{tabular}

3. بعد تحويل السلسلة إلى مصفوفة أحادية من الأحرف والحركات يتم تطبيق قوانين الفونولوجية العربية للوصول إلى ما يقابل الكتابة العروضية في الثعر العربي وكما يلي:

\begin{tabular}{|c|c|c|c|c|c|c|c|c|c|c|c|c|c|c|c|c|c|c|}
\hline$\delta$ & س & & & د & د & 8 & ب & & J & & b & b & $\delta$ & ب & & ت & $\delta$ & \\
\hline
\end{tabular}
4. إضافة السكون إلى الأحرف الغير محركة في النص:

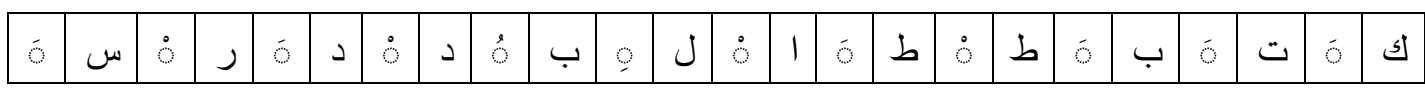

5. تكوين مصفوفة أحادية من الأرقام والتي تمثل شفرات الـ ASSCII) لكل من الحروف والحركات:

\begin{tabular}{|l|l|l|l|l|l|l|l|l|l|}
\hline 243 & 216 & 250 & 216 & 243 & 200 & 243 & 202 & 243 & 223 \\
\hline
\end{tabular}

\begin{tabular}{|l|l|l|l|l|l|l|l|l|l|l|}
\hline 209 & 243 & 207 & 250 & 207 & 245 & 200 & 246 & 225 & 250 & 199 \\
\hline
\end{tabular}

\begin{tabular}{|l|l|l|}
\hline 243 & 211 & 250 \\
\hline
\end{tabular}

6. تحويل كل حرف مع الحركة الخاصة به (الفونيم) إلى شفرة خاصة والتي تمثل أسم المقطع الخاص بالحرف (الألوفون) مع ملاحظة أن عملية الجمع تكون شفرة الحرف مع شفرة الحركة مضروبة في 100.

\begin{tabular}{|c|c|c|c|c|c|c|c|}
\hline 24700 & 24855 & 25199 & 24516 & 25216 & 24500 & 24502 & 24523 \\
\hline & & & & 24511 & 25209 & 24507 & 5207 \\
\hline
\end{tabular}

7. تكوين مصفوفة من السلاسل (Array of string) والتي تمثل أسماء الملفات الصوتية والتي تمثل الألفونات

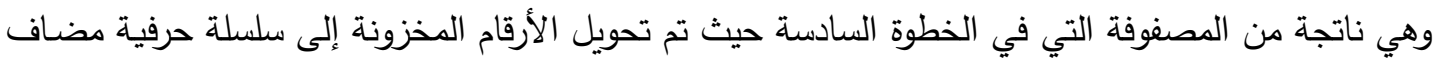
إليها المقطع ("WAV". ) : \begin{tabular}{|l|l|l|l|l|l|}
\hline 24511.WAV & 25209.WAV & 24207.WAV & 25207.WAV & 24700.WAV & 24855.WAV \\
\hline
\end{tabular}

\begin{tabular}{|l|l|l|l|l|l|}
\hline 25199.WAV & 24516.WAV & 25216.WAV & 24500.WAV & 24502.WAV & 24523.WAV \\
\hline
\end{tabular}


8. قراءة جميع الملفات الصوتية ودمجها في مصفوفة أحادية واحدة أممها (speech) ومن ثم يتم خزنها في ملف ولف الفي ثنائي أسمه (speech1.wav) والذي يمثل الملف الصوتي النهائي الذي سوف يتم تثغيله.

\section{"speech.wav"}

9.

7- الاستنتاجات:

1. هذا النظام كانت له القدرة على إنتاج عدد غير محدد من المفردات (كلمة، جملة) مع جودة عالية في الصوت.

2. تتمتع لغة (Visual Basic V.6) بمرونة عالية في التعامل مع النصوص والملفات الصوتية وتثغيلها

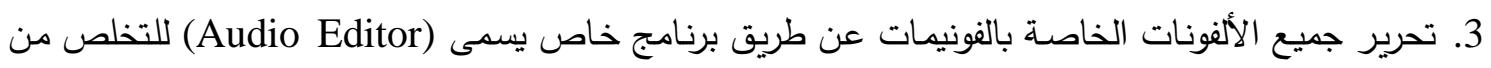

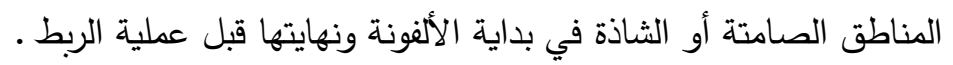
4. يجب تخزين الألفونات بمستوى واحد من سعة الموجة (Amplitude) لكي لا ينتج اختلاف في شدة الصوت لكل ألفونة ضمن الكلمة. 5. أن لكل حرف من الحروف العربية شفرة (ASSCII) خاص بها وكما هو معلوم، ومن حسن الحظ أن الحرف الأولي والوسطي والأخرى له نفس الثفرة مما سهل عملية تطبيق القوانين أعلاه. 6. يجب ربط الألفونات ببعضها البعض وتحويلها إلى ملف صوتي واحد ذي الامتداد (WAV.) لتجنب التقطع الحاصل من جراء تشغيل كل ألفونة لوحدها فيما لو لم يكن هنالك هذا الربط.

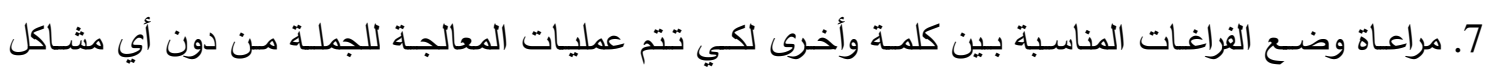
وتعقيدات برمجية. 8. لتطبيق قوانين الفونولوجية العربية يجب مراعاة التسلسل الوارد من المصدر المشار إليه في البحث لكي تتم

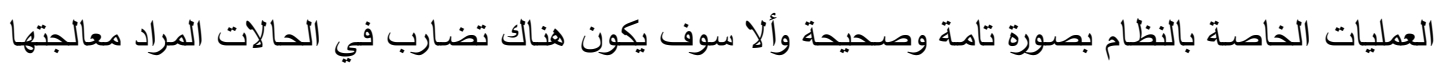
برمجياً. 9. إن بعض قوانين الفونولوجية العربية لم يتم تطبيقها من خلال البحث وذلك بسبب عدم الحاجة الملحة لها لبناء النظام 10. يمكن تطبيق هذا النظام على الأنظمة المطمورة. 11. هذا النظام الخاص باللغة العربية هو أكثر تعقيداً من الذي يستخدم مع اللغات الأخرى الأجنبية بسبب كون أن

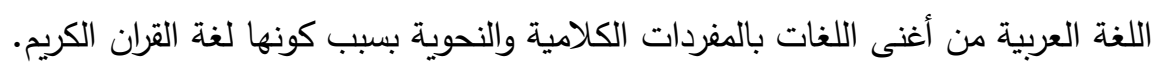
8- (التوصيات: بعد أنجاز البحث بنجاح نوصسي بتحسينه كإضـافة المصحح اللغوي والنحوي لتحليل الجمل المكتوبـة لغرض نطقها من ناحية القواعد الخاصـة باللغنة العربية حيث أن هذا العمل سوف يغني المستخدم نوعا مـا من نون تحريك الأحرف المكونة للجمل المراد نطقها. 


$$
\begin{aligned}
& \text { المصادر } \\
& \text { بدري، الدكتور كمال إبراهيم، (1988)، "علم اللغة المبرمج"، مطابع جامعة الملك سعود، الرياض. } \\
& \text { د. منصور محمد الغامدي، د. مصطفى الثـافعي أحمد، أ. حسني المحتسب، (1424)هـ، "نظام ألي } \\
& \text { لنطق النص العربي بالحاسب،جامعة الملك فهد للبترول والمعادن. }
\end{aligned}
$$

http://www.ccisdb.ksu.edu.sa/files/prs1280000.pptx

[3] Ghadeer Al-Said and Moussa Abdallah, (2009), "An Arabic Text-To-Speech System Based on Artificial Neural Networks", Journal of Computer Science 5.

[4] M. Z. Rashad, Hazem M. El-Bakry and Islam R. Isma'il, (2010), ”Diphone Speech Synthesis System for Arabic Using MARY TTS”, International journal of computer science \& information Technology (IJCSIT) Vol.2, No.4, August .

[5] Nader Abu Ghattas, and Hanna Abdel Nour, (2005), "Text-to-Speech Synthesis by Diphones for Modern Standard Arabic”, An-Najah Univ. J. Res. (N. Sc.), Vol. 19.

[6] O. Al-Dakkak, N. Ghneim, M. Abou Zliekha and S. Al-Moubayed, (2006), "Emotion Inclusion In An Arabic Text-To-Speech", http://www.w3.org/2006/02/SSML/papers/HIAST/final_Emotion.pdf.

[7] http://www.emaratesmac.com/formes/arabic. 


\begin{tabular}{|c|c|c|c|c|c|c|c|c|c|c|c|c|c|c|}
\hline \multicolumn{15}{|c|}{ A-Consonants: } \\
\hline $\begin{array}{l}\text { Arabic } \\
\text { Symbol }\end{array}$ & $\&$ & ب & ت & $\stackrel{\leftrightarrow}{\Delta}$ & ج & $\tau$ & $\dot{\tau}$ & د & $\dot{j}$ & J & j & س & ش & ص \\
\hline $\begin{array}{l}\text { Phonetic } \\
\text { Symbol }\end{array}$ & /?/ & /b/ & $/ t /$ & $\mid \theta /$ & $\mid 3 /$ & $/ \mathrm{h} /$ & $|x|$ & $/ \mathrm{d} /$ & /ð/ & $\mid \mathrm{r} /$ & $|z|$ & $/ \mathrm{s} /$ & 151 & $|\underline{\mathbf{s}}|$ \\
\hline $\begin{array}{l}\text { Arabic } \\
\text { Symbol }\end{array}$ & ض & b & ظ & $\varepsilon$ & $\dot{\varepsilon}$ & $\dot{0}$ & ت & s) & J. & s & $\dot{ن}$ & $\rightarrow$ & 9 & ي \\
\hline $\begin{array}{l}\text { Phonetic } \\
\text { Symbol }\end{array}$ & d d $/$ & $|\underline{t}|$ & / $\underline{\text { / }}$ & $|\varepsilon|$ & $|\gamma|$ & /fl & $|q|$ & $/ \mathrm{k}$ & /V & $/ \mathrm{m} /$ & $\ln /$ & $/ \mathrm{h} /$ & $|w|$ & $|j|$ \\
\hline
\end{tabular}

B- Vowels:

\begin{tabular}{|c|c|c|c|c|c|c|c|c|c|c|c|c|}
\hline $\begin{array}{l}\text { Arabic } \\
\text { Symbol }\end{array}$ & - & $\therefore$ & $\div$ & L & $r$ & $\longrightarrow$ & - & $\therefore$ & $\div$ & ᄂ & r & $\div$ \\
\hline $\begin{array}{l}\text { Phonetic } \\
\text { Symbol }\end{array}$ & $/ \mathrm{a} /$ & $/ \mathrm{u} /$ & /i/ & /aa/ & /uu/ & /iii/ & /â/ & $/ \hat{\mathrm{u}} /$ & $/ \hat{1} /$ & /ââ/ & /ûû/ & /î̀/ \\
\hline $\begin{array}{l}\text { Arabic } \\
\text { Symbol }\end{array}$ & $\therefore$ & $T$ & $r$ & & & & \multicolumn{6}{|c|}{ مفمة Pharyngealized } \\
\hline $\begin{array}{l}\text { Phonetic } \\
\text { Symbol }\end{array}$ & $10 /$ & $\mid \mathrm{e} /$ & $100 /$ & & & & & & & & & \\
\hline
\end{tabular}

